\title{
Peering at the outflow mechanisms in the transitional pulsar PSR J1023+0038: simultaneous VLT, XMM-Newton, and Swift high-time resolution observations ${ }^{\star}$
}

\author{
M. C. Baglio ${ }^{1,2}$, F. Vincentelli ${ }^{3,4,2}$, S. Campana ${ }^{2}$,F. Coti Zelati ${ }^{5,2}$, P. D’Avanzo ${ }^{2}$, L. Burderi ${ }^{6}$, P. Casella ${ }^{4}$ \\ A. Papitto ${ }^{4}$, and D. M. Russell ${ }^{1}$ \\ 1 Center for Astro, Particle and Planetary Physics, New York University Abu Dhabi, PO Box 129188, Abu Dhabi, UAE \\ e-mail: mcb19@nyu.edu \\ 2 INAF, Osservatorio Astronomico di Brera, Via E. Bianchi 46, 23807 Merate, LC, Italy \\ 3 Department of Physics \& Astronomy, University of Southampton, Highfield, Southampton SO17 1BJ, UK \\ 4 INAF, Osservatorio Astronomico di Roma, Via Frascati 33, 00078 Monteporzio Catone, Roma, Italy \\ 5 Institute of Space Sciences (ICE, CSIC-IEEC), Campus UAB, Carrer Can Magrans s/n, 08193 Barcelona, Spain \\ ${ }^{6}$ Università degli Studi di Cagliari, Dipartimento di Fisica, SP Monserrato-Sestu, km 0.7, 09042 Monserrato, CA, Italy
}

Received 3 June 2019 / Accepted 11 September 2019

\begin{abstract}
We report on a simultaneous near-infrared, optical, and X-ray campaign performed in 2017 with the XMM-Newton and Swift satellites and the HAWK-I instrument mounted on the Very Large Telescope (VLT) on the transitional millisecond pulsar PSR J1023+0038. Near-infrared observations were performed in fast-photometric mode $(0.5 \mathrm{~s}$ exposure time $)$ in order to detect any fast variation of the flux and correlate this with the optical and X-ray light curves. The optical light curve shows the typical sinusoidal modulation at the system orbital period $(4.75 \mathrm{~h})$. No significant flaring or flickering is found in the optical, nor any signs of transitions between active and passive states. On the contrary, the near-infrared light curve displays a bimodal behaviour, showing strong flares in the first part of the curve, and an almost flat trend in the rest. The X-ray light curves instead show a few low-high mode transitions, but no flaring activity is detected. Interestingly, one of the low-high mode transitions occurs at the same time as the emission of an infrared flare. This can be interpreted in terms of the emission of an outflow or a jet: the infrared flare could be due to the evolving spectrum of the jet, which possesses a break frequency that moves from higher (near-infrared) to lower (radio) frequencies after the launching, which has to occur at the low-high mode transition. We also present the cross-correlation function between the optical and near-infrared curves. The near.infrared curve is bimodal, therefore we divided it into two parts (flaring and quiet). While the cross-correlation function of the quiet part is found to be flat, the function that refers to the flaring part shows a narrow peak at $\sim 10 \mathrm{~s}$, which indicates a delay of the near-infrared emission with respect to the optical. This lag can be interpreted as reprocessing of the optical emission at the light cylinder radius with a stream of matter spiraling around the system due to a phase of radio ejection. This strongly supports a different origin of the infrared flares that are observed for PSR J1023+0038 with respect to the optical and X-ray flaring activity that has been reported in other works on the same source.
\end{abstract}

Key words. stars: jets - stars: neutron $-\mathrm{X}$-rays: binaries

\section{Introduction}

Millisecond pulsars (MSPs) are neutron stars (NSs) that emit pulsed radiation (typically in the radio band, but also in the $\mathrm{X}$ - and $\gamma$-rays) with a period within the 1-10 milliseconds range. According to the recycling scenario, MSPs are NSs that were originally hosted in a binary system (typically a low-mass X-ray binary, or LMXB) that have been re-accelerated through accretion. In particular, the angular momentum transfer due to accretion could increase the rotational velocity of the NS around its axis by up to one hundred times per second or more, as observed in MSPs (Alpar et al. 1982; Radhakrishnan \& Srinivasan 1982; Srinivasan 2010).

The recycling scenario of MSPs was first confirmed with the discovery of millisecond X-ray pulsations from the NS LMXB SAX J1808.4-3658 (Wijnands \& van der Klis 1998), and more recently with the so-called missing link pulsar PSR J1023+038

\footnotetext{
$\star$ Tables of the light curves are only available at the CDS via anonymous ftp to cdsarc.u-strasbg.fr $(130.79 .128 .5)$ or via http: //cdsarc.u-strasbg.fr/viz-bin/cat/J/A+A/631/A104
}

(hereafter J1023), which was the first source that was discovered to transit between a radio pulsar state and an LMXB state (Archibald et al. 2009). This was the first source to be classified as a transitional millisecond pulsar (tMSP). The first system that instead showed a full outburst cycle, from a bright X-ray phase $\left(\sim 10^{36} \mathrm{erg} \mathrm{s}^{-1}\right)$ to quiescence, was IGR J18245-2452 (Papitto et al. 2013). Nearly two weeks after it settled to quiescence, a radio pulsar began to shine, which confirmed the MSP nature of the compact object.

J1023 was first detected by Bond et al. (2002) in the radio band, and it later showed clear signatures of an accretion disc around the compact object in the optical (Szkody et al. 2003). Thorstensen \& Armstrong (2005) later identified J1023 as an NSLMXB. Observations carried out in 2003-2005 revealed no presence of the accretion disc, however: the spectrum was consistent with that of a G-type star, but signatures of strong irradiation were instead detected (Thorstensen \& Armstrong 2005). In 2007, the compact object of the system was identified as a 1.69 ms period radio pulsar (Archibald et al. 2013). Finally, J1023 underwent a state change from the MSP state to an LMXB state 
(where it has remained until 2019) in June 2013, and the radio pulsar signal consequently switched off (Stappers et al. 2014). A large number of multi-wavelength studies have been performed on this source since its last state transition (see e.g. Takata et al. 2014; Tendulkar et al. 2014; Coti Zelati et al. 2014, 2018; Deller et al. 2015; Bogdanov et al. 2015, 2018; Shahbaz et al. 2015, 2018; Baglio et al. 2016a; Campana et al. 2016; Jaodand et al. 2016; Ambrosino et al. 2017; Papitto et al. 2018).

In the LMXB state, the source reveals a very peculiar behaviour in the X-rays (Bogdanov et al. 2015): J1023 exhibits frequent mode switchings between three different X-ray states. For $\sim 70 \%$ of the time, a high luminosity mode $\left(L_{X} \sim 7 \times\right.$ $10^{33} \mathrm{erg} \mathrm{s}^{-1}, 0.3-79 \mathrm{keV}$; Tendulkar et al. 2014) occurs, which is characterized by the observation of coherent X-ray pulsations (Archibald et al. 2015), with an rms pulsed fraction of $\sim 8 \%$. For $20 \%$ of the time, the system is in a low luminosity mode, characterized by $L_{X} \sim 5 \times 10^{32} \mathrm{erg} \mathrm{s}^{-1}$ and by the absence of $\mathrm{X}$-ray pulsations. Finally, for $\sim 2 \%$ of the time, a flaring mode occurs, during which a sudden increase in X-ray luminosity up to $3 \times 10^{34} \mathrm{erg} \mathrm{s}^{-1}$ is reported (Bogdanov et al. 2015). Luminosities are calculated at a distance of $1.37 \mathrm{kpc}$, as derived from radio parallax (Deller et al. 2015). Transitions between the highand low- luminosity modes occur on a timescale of $\sim 10 \mathrm{~s}$, and are visualized in the X-ray light curve as rectangular shapes. Similarly, at optical frequencies frequent transitions between two different modes (named active and passive mode) were first observed by Shahbaz et al. (2015) as rectangular dips in the light curves, with ingress and egress times of $\sim 20 \mathrm{~s}$. However, the existence of a mode-switching at optical frequencies is currently strongly debated. Bright emission in the radio with a flat to slightly inverted spectrum was detected by Deller et al. (2015) and was interpreted as due to the emission of a compact self-absorbed jet. Bogdanov et al. (2018) recently observed the emission of radio flares in correspondence with the X-ray low mode, while during the high mode, a steady radio emission was detected. This strong anti-correlation between the radio and the $\mathrm{X}$-ray light curve was again interpreted as due to synchrotron emission from jets or outflows. Optical phase-resolved linear polarization measurements were also performed (Baglio et al. 2016a; Hakala \& Kajava 2018), revealing a degree of polarization of a few percent, which is better interpreted as due to Thomson scattering from the accretion disc than to synchrotron emission from a jet. Strong flickering and flaring activity was also observed in the near-infrared (NIR; Hakala \& Kajava 2018; Shahbaz et al. 2018) and was interpreted as due to reprocessing of the optical emission and to a combination of reprocessing and emission from plasmoids in the accretion flow in the NIR. In addition, Kepler monitoring found a much stronger flaring activity in the optical than in the X-ray band (see Papitto et al. 2018).

Very recently, optical pulsations at the pulsar spin period were observed during the accretion state, making J1023 the first optical millisecond pulsar ever detected (Ambrosino et al. 2017; see also Zampieri et al. 2019). This puzzling behaviour was interpreted as due to an active rotation-powered millisecond pulsar in the system.

Several models have been proposed in order to explain the unprecedented variety of features observed for this system. Some of these models involve an active pulsar that is enshrouded by the accretion disc or by a plasma of ionized matter, causing the interaction between the pulsar wind and the ionized matter and therefore the emission of synchrotron radiation in the X-rays (Takata et al. 2014; Coti Zelati et al. 2014; Li et al. 2014); others see a fast-spinning NS that propels the matter from the accretion disc away (Papitto \& Torres 2015); still others instead consider the alternation between a radio pulsar and a propeller regime, which might account for the continuous switches between the low and high X-ray modes (Campana et al. 2016; Coti Zelati et al. 2018). Recently, Papitto et al. (2019) suggested that J1023 might be a rotation-powered pulsar emitting a magnetized wind that interacts with the accreting mass close to the light cylinder radius and thus creates a shock. At this shock, electrons are accelerated, producing optical and X-ray pulsations that have a synchrotron nature. This interpretation also accounts for the lack of pulsations in the X-ray and optical in the low mode by hypothesizing that the shock is moved at larger distance by the pulsar wind, and of the flaring mode, considering that the shock region might increase its size.

In this paper we show the results of a simultaneous NIR, optical, and X-ray fast-imaging campaign performed with the Very Large Telescope (VLT), XMM-Newton, and Swift on PSR J1023+0038 on 2017 June 9-10. Fast NIR photometry was performed simultaneously to X-ray observations, which allowed us to link the fast variability of the NIR light curve to variations in the X-ray (and optical) emission. The details of the observation and the data analysis are reported in Sect. 2, in Sect. 3 the main results of the campaign are shown, and the discussion and conclusions are drawn in Sects. 4 and 5.

\section{Observation and data analysis}

\subsection{Near-infrared fast photometry}

We collected NIR ( $J$-band, $1.2 \mu \mathrm{m}$ ) high time-resolution data with the HAWK-I instrument mounted on the VLT, UT-4/Yepun, on 2017 June 10 . HAWK-I is a NIR wide-field imager (0.97 to $2.31 \mu \mathrm{m}$ ) made by four HAWAII 2 RG $2048 \times 2048$ pixels detectors displayed in a $2 \times 2$ configuration (Kissler-Patig et al. 2008). Each quadrant has a field of view (FoV) of $217 \operatorname{arcsec}^{2}$ and is separated by the nearby detector by $15^{\prime \prime}$ gap. The total FoV is therefore $7.5^{\prime} \times 7.5^{\prime}$ In order to reach sub-second time resolutions, observations were performed in Fast Phot mode: this mode limits the area on which the detector is read to one stripe in each quadrant. In particular, we set the instrument to read 16 contiguous windows of $128 \times 256$ pixels. HAWK-I was pointed to (RA, Dec) 10:23:57.0, +00:37:34.3 with a rotation angle of $-17.8^{\circ}$ in order to place the source and a reference star (with a known Vega $J$-band magnitude of $13.51 \pm 0.03$ ) in the upper right quadrant (Q3). During the observation, a series of 100 frames were stacked together and formed a "data-cube": each frame has a time resolution of $0.5 \mathrm{~s}$. A gap of $\sim 3 \mathrm{~s}$ is present between each cube. Photometric data were extracted using the ULTRACAM data reduction software tools (Dhillon et al. 2007). For the light-curve extraction, an aperture radius of $1.3^{\prime \prime}$ was used, while for the background we used an annulus that extended from $1.9^{\prime \prime}$ to $2.7^{\prime \prime}$ around the source. Parameters for the extraction were derived from the reference star (RA, Dec $=10: 23: 43.31$, $+00: 38: 19.1)$ and position. To account for seeing effects, the ratio between the source and the reference star count rate was used. The time of each frame was then placed into the barycentric dynamical time system, and the photon arrival times were referred to the solar system barycenter.

\subsection{X-ray observations}

\subsubsection{XMM-Newton observations}

XMM-Newton observed J1023 on 2017 June 9. Because of a technical problem, no data were gathered from the pn camera. 


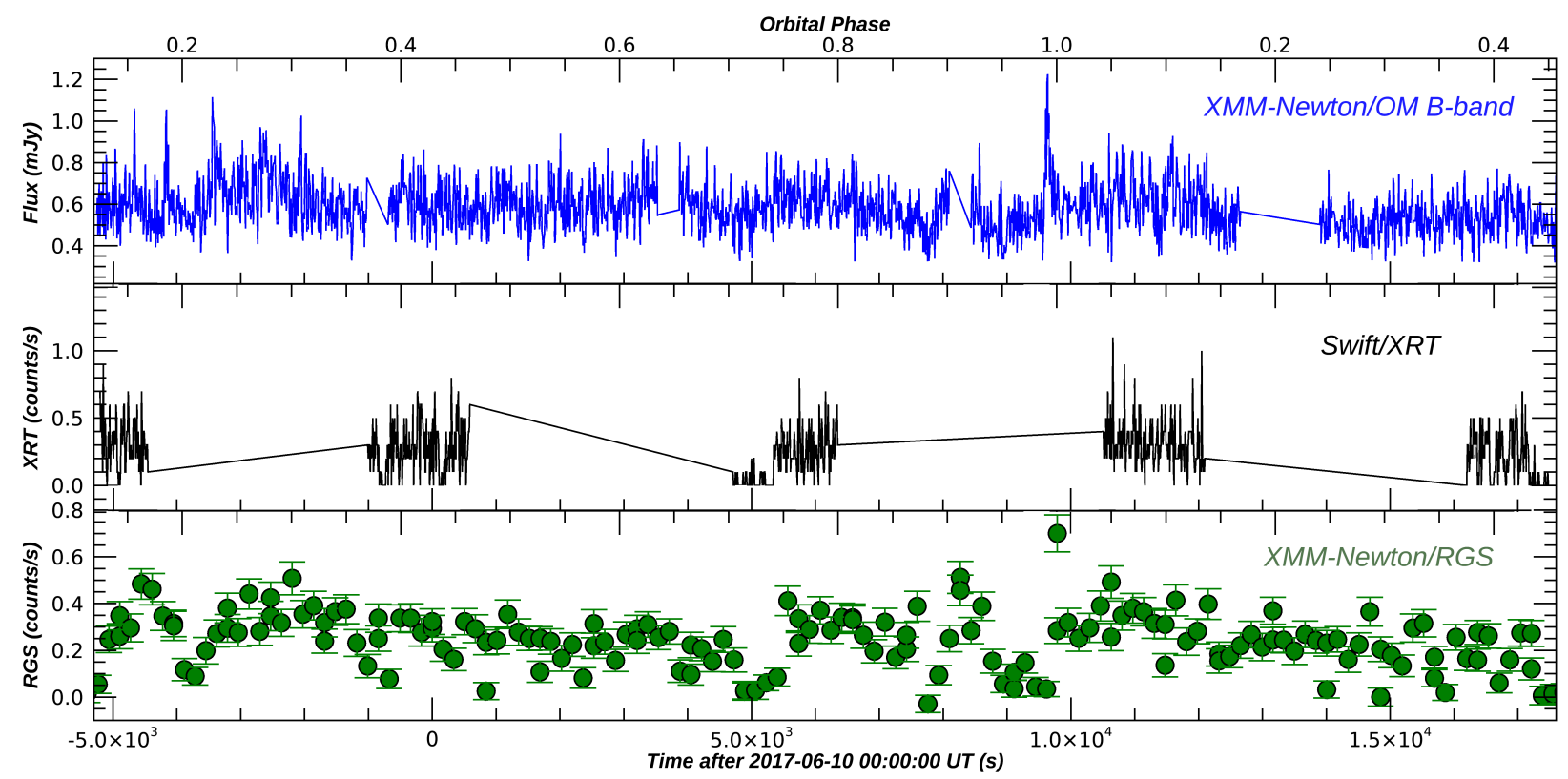

Fig. 1. From top to bottom, B-band (OM), Swift/XRT, and XMM-Newton RGS light curves of PSR J1023 (bin time of 10 s). The optical light curve has been de-trended. Errors are reported at the 68\% confidence level and are shown for the RGS light curve only. For the OM and the Swift/XRT curve, errors (68\% confidence level) are of the order of $0.1 \mathrm{mJy}$ and 0.2 counts s$^{-1}$, respectively. The bin time is $10 \mathrm{~s}$ for the $B$-band and the Swift/XRT curves, and 140 s for the XMM-Newton RGS curve. The top $x$-axis reports the orbital phases of the system, which have been evaluated starting from the ephemeris of Archibald et al. (2009), phase 0 being the inferior conjunction of the companion star.

The MOS1 camera (in small-window mode) was exposed for $1.3 \mathrm{ks}$, and the MOS2 camera (in small-window mode and overlapping with MOS1 data) for $10 \mathrm{ks}$, out of $124 \mathrm{ks}$ requested, owing to anomalies of the instruments during the observation. MOS2 data were collected only for the first $420 \mathrm{~s}$, however. We do not consider EPIC data in the following.

The RGSs (den Herder et al. 2001) were configured in standard spectroscopy mode (in the $0.35-2 \mathrm{keV}$ energy range) and observed the target for $\sim 24 \mathrm{ks}$. We processed the data of each RGS instrument using the task rgsproc, and extracted a combined RGS1 and RGS2 first- and second-order exposure-corrected and background-subtracted light curve using rgslccorr. We adopted a time bin of $140 \mathrm{~s}$ so as to single out the high and low X-ray modes.

\subsubsection{Swift/XRT observations}

Swift/XRT started observing J1023 on 2017 June 9 at 22:34:04. The XRT was operated in photon-counting mode $(2.5 \mathrm{~s}$ time resolution). The observation lasted $7.0 \mathrm{ks}$, split into five consecutive orbits. Data were processed with xrtpipeline to derive a cleaned event file. A light curve in the $0.3-10 \mathrm{keV}$ energy band was extracted from a circular region with a radius of 35 pixels. The source count rate is $\sim 0.3$ counts $\mathrm{s}^{-1}$. The background count rate amounts to $1.4 \%$ of the source rate. The UVOT was operated in image mode with the $m 2$ filter. These data are not considered here.

\subsection{Optical observations}

The XMM-Newton Optical Monitor (OM) observed J1023 starting on June 9 at 22:32:27 UTC in fast mode, using the $B$ filter (central effective wavelength of $450 \mathrm{~nm}$ ). Data were taken into five subsequent exposures lasting $4387.5 \mathrm{~s}$ each in fast mode (0.11 s time resolution). The data set ended on 2017 June 10, 05:02:13, well before the expected end. Data were processed using omfchain. J1023 is well detected with a backgroundsubtracted mean count rate of $\sim 7$ count $\mathrm{s}^{-1}$.

\section{Results}

\subsection{Light curves}

In Figs. 1 and 2, the simultaneous NIR (HAWK-I $J$-band), optical (XMM-Newton OM B-band) and X-ray (Swift/XRT and XMM-Newton RGS) light curves of J1023 are presented. We calculated the orbital phases based on the ephemeris of Archibald et al. (2009). Phase 0 corresponds to the inferior conjunction of the companion, that is, when the observer sees the non-irradiated face of the donor star. The sinusoidal modulation (with a semi-amplitude of $\sim 9-10 \%$ ) at the known $4.75 \mathrm{~h}$ orbital period of the source was de-trended from the original $B$-band light curve, leaving an optical curve that still shows signs of erratic variability (Fig. 1, upper panel). The $J$-band light curve does not clearly show any sinusoidal variability at the system orbital period (Fig. 2, top panel), consistent with previous works on the source at NIR wavelengths (see Coti Zelati et al. 2014; Shahbaz et al. 2018). The expected $J$-band curve semiamplitude, considering our $B$-band measured semi-amplitude and the values reported in Coti Zelati et al. (2014) as comparison, is $\sim 5 \%$, which is of the order of the error on the single flux measurement reported in our $J$-band light curve. We therefore conclude that any sinusoidal modulation of our $J$-band light curve is likely hidden in the intrinsic statistical uncertainty of our data. Instead, a frequent flaring activity is observed, superimposed on an overall constant behaviour of the curve. This is reminiscent of what is observed in the NIR by Shahbaz et al. (2018) and Papitto et al. (2019).

We note that the strong infrared flaring activity is mostly concentrated in the first $\sim 5000 \mathrm{~s}$ of observation. The second part of the light curve shows a more quiet behaviour (Fig. 2, upper panel). 


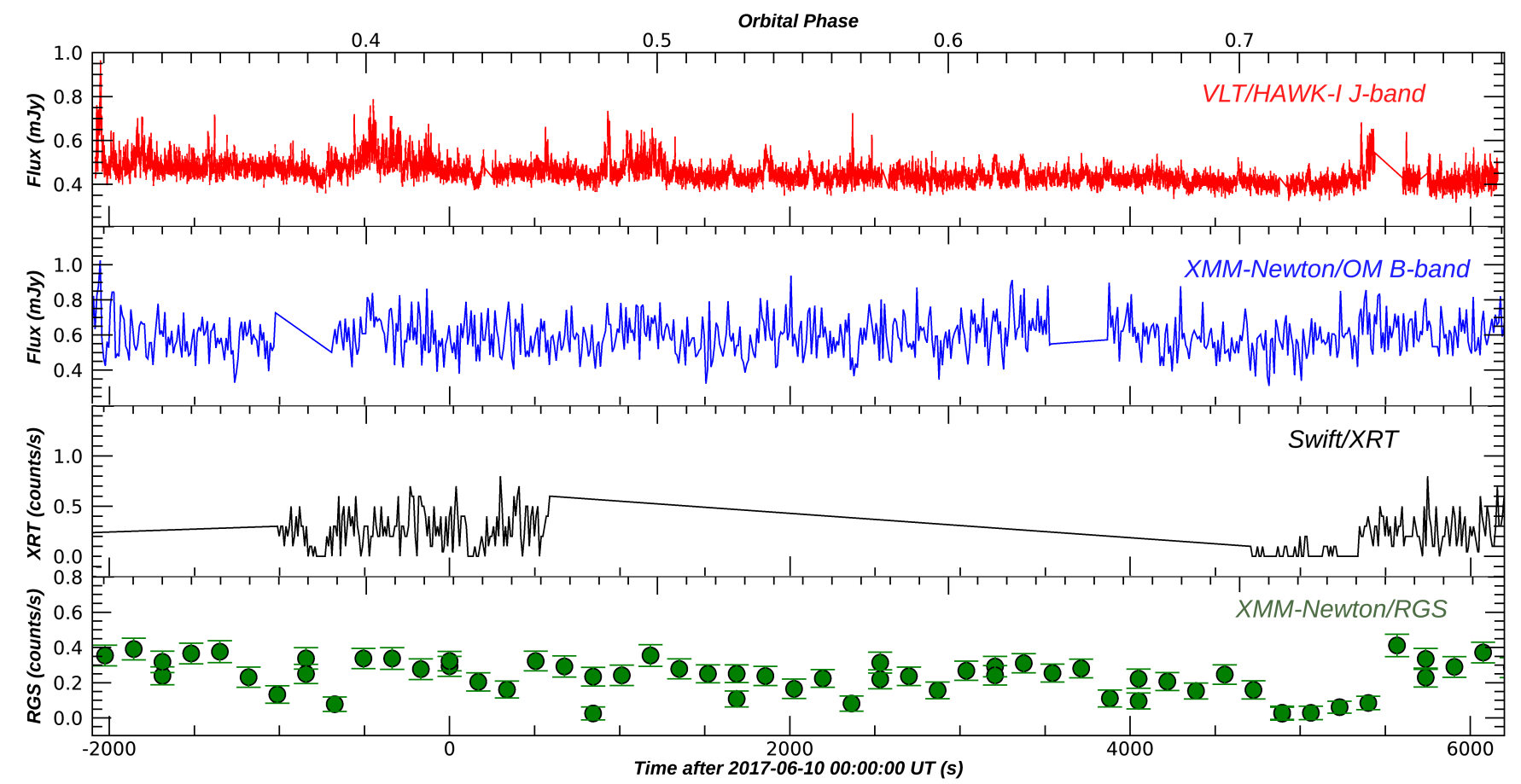

Fig. 2. From top to bottom, J-band (HAWK-I), B-band (OM), Swift/XRT, and XMM-Newton RGS light curves of PSR J1023. The bin time is $0.5 \mathrm{~s}$ for the $J$-band curve, $10 \mathrm{~s}$ for the $B$-band and the Swift/XRT curves, and $140 \mathrm{~s}$ for the XMM-Newton RGS curve. The optical light curve has been de-trended. Errors are reported at the $68 \%$ confidence level and are shown for the RGS light curve only. For the HAWK-I, OM, and the Swift/XRT curves, errors (68\% confidence level) are of the order of $0.02 \mathrm{mJy}, 0.1 \mathrm{mJy}$, and 0.2 counts s$^{-1}$, respectively. The top $x$-axis reports the orbital phases of the system, which have been evaluated starting from the ephemeris of Archibald et al. (2009), phase 0 being the inferior conjunction of the companion star.

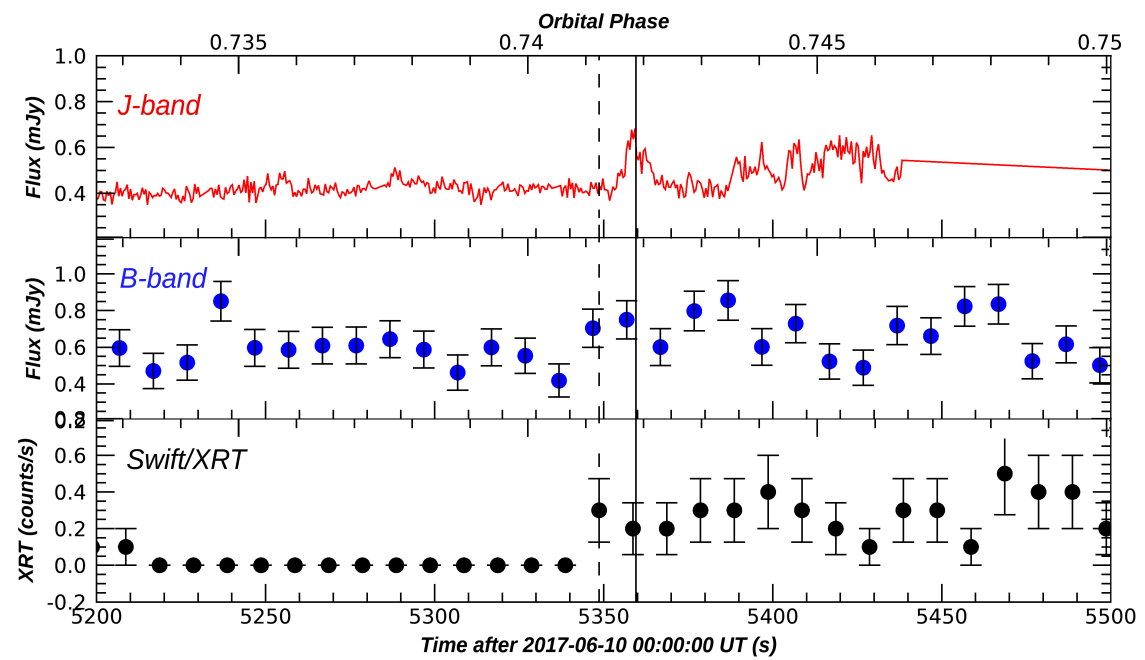

Fig. 3. From top to bottom, zoom of the NIR, optical, and X-ray (Swift/XRT) light curves of J1023 around the time of the X-ray low-high transition. With the dashed black line we indicate the exact time of the transition and with the solid black line we show the time corresponding to the centroid of the NIR flare. The top $x$-axis reports the orbital phases of the system, which have been evaluated starting from the ephemeris of Archibald et al. (2009), phase 0 being the inferior conjunction of the companion star.

The Swift/XRT light curve shown in the central panel of Fig. 1 is almost featureless, except for one transition from the low to the high mode at $\sim 5000 \mathrm{~s}$ after 2017-06-10 00:00:00 UT. In Fig. 3, a zoom of the three light curves around the time of the X-ray low-high transition is shown. In relation to the lowhigh transition, we observe an almost simultaneous increase in the optical flux by a factor of 1.7 in $10 \mathrm{~s}$ (Fig. 3, middle panel), and the emission of a flare in the NIR. To evaluate the time lag between the two, we considered the time corresponding to the first X-ray point associated with the high mode as the time of the low-high transition, and the centroid of the Gaussian function that best fits the NIR flare as the time of the emission of the flare. In this way, a lag of $11 \pm 5 \mathrm{~s}$ was estimated (Fig. 3, top panel), with the infrared delayed with respect to the X-rays.
To have a better understanding of the origin of the $J$-band flare in correspondence to the low-high transition, we evaluated the slope $\alpha$ of the optical to NIR spectral energy distribution (SED). We defined the slope of the SED starting from the $B$-band and $J$-band fluxes $\left(F_{B}\right.$ and $F_{J}$, respectively) as follows:

$\alpha=\frac{\log _{10}\left(F_{B}\right)-\log _{10}\left(F_{J}\right)}{\log _{10}\left(v_{B}\right)-\log _{10}\left(v_{J}\right)}$,

where $v_{B}$ and $v_{J}$ are the central frequencies of the $B$-band $(6.67 \times$ $\left.10^{14} \mathrm{~Hz}\right)$ and $J$-band $\left(2.43 \times 10^{14} \mathrm{~Hz}\right)$ filters. Fluxes were dereddened using the coefficients reported in Baglio et al. (2016a).

The slope of the SED stays approximately constant during the whole duration of our observations (Fig. 4), with a slow 


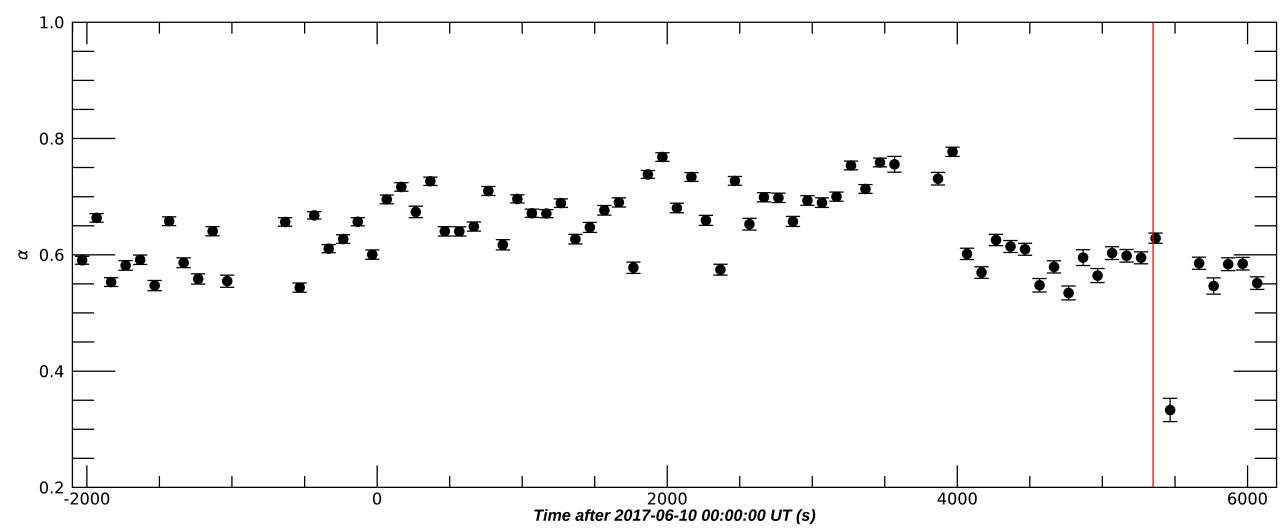

Fig. 4. Trend with time of the slope $\alpha$ of the optical and NIR spectral energy distribution of PSR J1023, defined as in Eq. (1). The solid red line indicates the exact time of the low-high X-ray transition. The bin time of the curve is $100 \mathrm{~s}$. Errors are represented at the $68 \%$ confidence level.

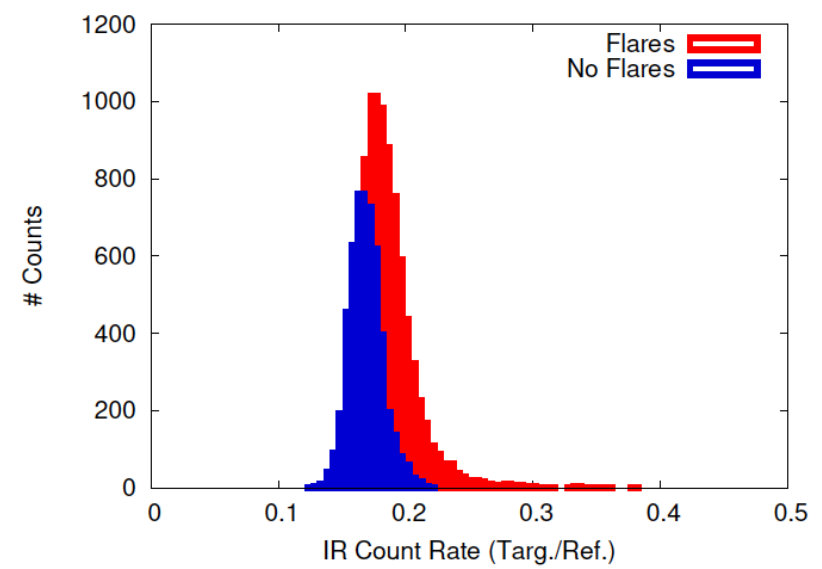

Fig. 5. Near-infrared light-curve distribution of the two regimes. In red we present the first more variable segment and in blue we plot the most stable segment. Gaussian fits of the two distributions give reduced $\chi^{2}$ of 1.2 and 4.4 in the quiet and flare part of the light curve with 19 and 29 degrees of freedom, respectively, in accordance with the asymmetry of the flaring part distribution, which is also visible by eye.

increase in the index before $4000 \mathrm{~s}$ and a sudden drop after $4000 \mathrm{~s}$. No clear variation of the slope in proximity to the infrared flare is detected, however.

\subsection{Correlations}

With the aim of revealing any correlation between the NIR and optical, we tried to evaluate the cross-correlation function (CCF) between the two (NIR and optical) light curves. To do this, only strictly simultaneous optical and NIR data were considered. The time resolution of the optical light curve is lower than the NIR ( $10 \mathrm{~s}$ vs. $0.5 \mathrm{~s}$, respectively), therefore we decided to re-bin the NIR light curve to a $10 \mathrm{~s}$ time resolution to evaluate the CCF.

The OM light curve shows strong flares at the beginning and end of the exposure; the NIR light curve instead has a nonstationary behaviour. As shown in Fig. 2 (top panel), the first $\sim 5000 \mathrm{~s}$ of the NIR light curve are characterized by strong and structured variability, while the last half of the observation does not show signs of strong variability, except for the flare in correspondence to the low-high mode transition at $\sim 5000 \mathrm{~s}$ after 10/06/2017 00:00:00 UT (Fig. 3, top panel).

This difference is also evident when we compute the distribution of the fluxes for the NIR light curve, that is, the histogram of the $J$-band count-rate values. We computed the histogram of the light curve in these two different regimes: first, we used the first $\sim 5000 \mathrm{~s}$ of observation, characterized by strong and structured

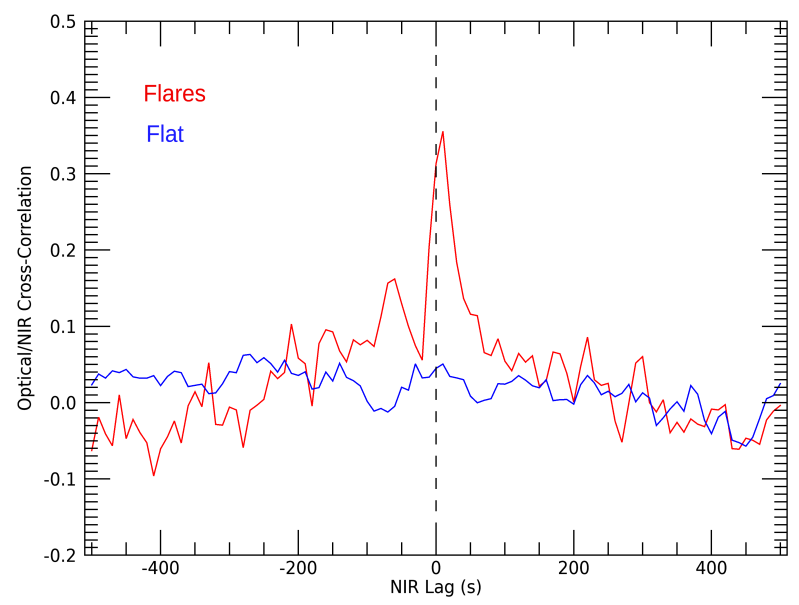

Fig. 6. Optical to NIR CCF computed in two different selected segments of the NIR light curve. A strong peak is present only in the variable part of the light curve. Uncertainties are of the order of 0.05 (68\% confidence level).

variability; secondly, we considered the $\sim 1000$ s before the lowhigh X-ray transition, where the light curve is almost flat, without signs of variability. In the first case, an asymmetric distribution was found, with a long tail towards high fluxes that is clearly due to the structured variability. In the second, the distribution is instead symmetric and can be well fitted with a log-normal distribution (with a reduced $\chi^{2}$ of 1.02; see Eq. (3) in Uttley et al. 2005). The two distributions are shown in Fig. 5.

Because of this bimodality of the NIR light curve, we decided to compute the optical to NIR CCF using these two separated segments (Fig. 6). While the second CCF, which is referred to as the flat part of the light curve (represented in blue in Fig. 6), does not show any significant feature, the first CCF (in red in Fig. 6) presents a clear peak at $7.0 \pm 1.3 \mathrm{~s}$. This is an indication that at least for the part of the light curve that shows flares, the NIR emission has a delay of $\sim 10 \mathrm{~s}$ with respect to the optical.

A similar result was also achieved in Shahbaz et al. (2018), where a narrow, positive peak at $\sim 5 \mathrm{~s}$ was detected in the CCF between the optical $r^{\prime}$ band and the NIR $K$ band. However, they also detected a broad anti-correlation on a timescale of $\sim-300 \mathrm{~s}$ and a broad positive correlation at $+300 \mathrm{~s}$, which we do not observe in our CCF.

\subsection{Power density spectrum}

The NIR power density spectrum (PDS) was computed using 128 bin per segment and a logarithmic binning factor of 1.05 , 


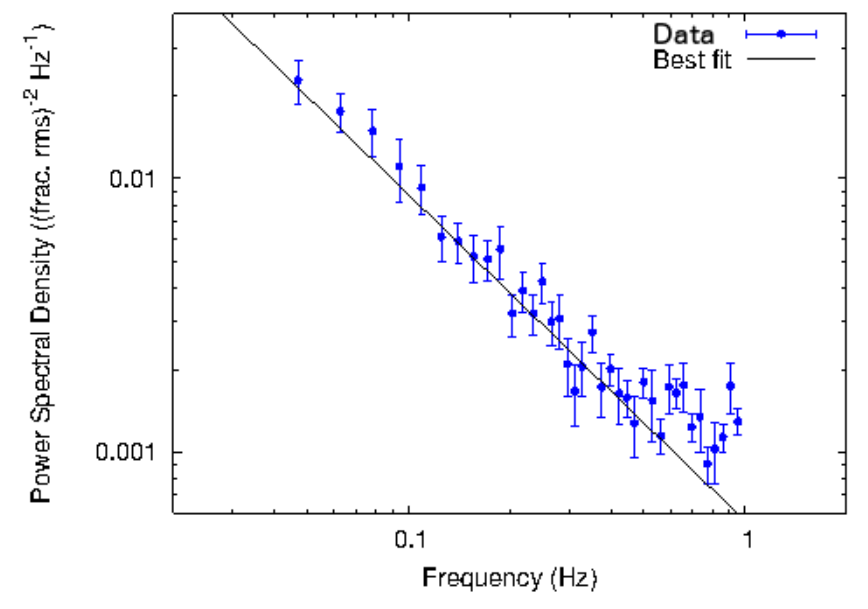

Fig. 7. Power density spectrum of the $J$-band light curve of PSR J1023+0038. Superimposed we show the power-law fit to the PDS (solid line).

allowing us to probe frequencies from 0.0625 to $1 \mathrm{~Hz}$. The maximum frequency probed in this work is a factor of ten higher than the most recent study of the IR fast variability of this source (Shahbaz et al. 2018). The PDS shows a decreasing power-law trend with increasing frequencies (Fig. 7), and seems to become flatter for higher frequencies. Following Shahbaz et al. (2018), we performed a fit between 0.02 and $0.2 \mathrm{~Hz}$ with a power-law model. The best fit returned an index of $-1.23 \pm 0.08$, which is consistent with the slope measured by Shahbaz et al. (2018), and also with the slope measured in the optical by Papitto et al. (2018), in the UV by Hernandez Santisteban (2016) and in the X-rays by Tendulkar et al. (2014), within the uncertainties. As shown in Fig. 7, there is an excess in the PDS for frequencies higher than $0.6 \mathrm{~Hz}$. However, given the signal near the Nyquist frequency, this excess is spurious and can be explained in terms of aliasing (i.e. the reflection of the power that is present above the Nyquist frequency also at the frequencies below it; see van der Klis 1988 for a review).

This result indicates an aperiodic activity and is typically observed in X-ray binaries, both in outburst and quiescence, for which PDS indexes in the range -1 to -2 have been measured in the infrared (see Shahbaz et al. 2018 and references therein).

\section{Discussion}

\subsection{Origin of the $\sim 10$ s NIR to optical lag}

Studies of the correlations between light curves at different wavelengths are an extremely powerful tool for determining the physical processes that are taking place. In particular, crosscorrelation studies allow us to determine any delay between light curves at different frequencies. Fast-photometry observations are in this sense crucial when processes are investigated that occur on short timescales. With our campaign, we obtained NIR-infrared ( $J$-band) observations of PSR J1023+0038 with an integration time of $0.5 \mathrm{~s}$ simultaneously with $X M M-N e w t o n / \mathrm{OM}$, Swift/XRT, and XMM-Newton RGS observations.

Cross-correlation studies of PSR J1023 have already been performed and published. In particular, Shahbaz et al. (2018) reported results of an NIR ( $K$-band) and optical ( $r^{\prime}$-band) campaign that revealed a strong broad anti-correlation at negative lags and a broad positive correlation at positive lags, with a superimposed narrow correlation. They interpreted their results as due to reprocessing of the optical emission at infrared frequencies, the narrow positive lag being peaked at $\sim 5 \mathrm{~s}$, which is consistent with the timescale for the light travel between the binary components, considering a binary separation of $\sim 1.8 R_{\odot}$. Moreover, the resolution of their optical light curve was good enough for them to observe several active/passive mode transitions, as were previously reported to occur in the optical by Shahbaz et al. (2015).

Recently, an X-ray, optical, and NIR timing study on the source has been performed (Papitto et al. 2019). In this work, the optical and X-ray correlation has been extensively studied, and the CCFs were evaluated during the X-ray flaring, high, and low modes at timescales ranging from 1 to $2.5 \mathrm{~s}$ over $64 \mathrm{~s}$ intervals. $\mathrm{X}$-ray and optical emissions are correlated in all three modes, the optical variability being always delayed with respect to the X-ray variability. Papitto et al. (2019) also investigated the connection between NIR and X-ray emission. The two curves were found to be correlated, with the emission of a slightly lower infrared flux during the X-ray low mode with respect to the high mode. Moreover, they also found evidence that infrared flares occur after the transition from the low to the high X-ray mode in three cases, but they did not observe a corresponding flare at the start of any X-ray low mode.

Because the time resolution and quality of our datasets are different, a comparison between our results and those reported in Shahbaz et al. (2018) is difficult to perform. When the orbital modulation is subtracted (Fig. 1), we do not observe any transition from the active to the passive mode (and vice versa) in our $B$-band light curve, in which only erratic variability is detected. Some flares are also observed, but the lack of any simultaneous observation at a different wavelength makes an interpretation hard. The results reported in Fig. 9 of Shahbaz et al. (2018) might explain the absence of any transition from active to passe mode in our light curve. According to their analysis, the expected flux difference between the active and passive mode is supposed to vary depending on the wavelength of the observation, being more pronounced in the $r^{\prime}$ band (i.e. the band in which Shahbaz et al. 2018 observed) than in our $B$ band. In particular, we should expect a variation of $\sim 0.1 \mathrm{mJy}$ in the $B$ band that is comparable with the dimension of the error bars in our light curve (see Fig. 3, middle panel). Therefore, it is almost impossible to detect any transition from active to passive mode in our optical light curve. In addition, the CCF that we built between our NIR and optical light curves (Fig. 6) does not show many similarities with the light curve reported in Shahbaz et al. (2018). In particular, we observe for the portion of the light curve where NIR flares are detected (red curve in Fig. 6) a narrow positive lag of the NIR with respect to the optical at $7.0 \pm 1.3 \mathrm{~s}$, which is far higher (almost double) than the one observed in Shahbaz et al. (2018). Moreover, we do not observe any broad correlation or anti-correlation in this CCF. The blue curve in Fig. 6, that is, the curve that refers to the part of the light curve without flares, is instead completely featureless, meaning that no correlation can be found between these portions of the optical and NIR light curves.

When we first focus on the flat parts of the light curves (blue curve in Fig. 6), we can easily convert the values of the fluxes into magnitudes, and calculate a mean value of $15.430 \pm 0.030$, which is consistent with the mean magnitude in the $J$ band reported in the literature for PSR J1023 (see e.g. Coti Zelati et al. 2014; Baglio et al. 2016a). In particular, Baglio et al. (2016a) reported the broadband fit of the NIR-X-ray SED, demonstrating that the NIR and optical part of the curve can be well fitted by a combination of the contribution of an irradiated star (which 
is predominant in the $J$ band) plus the truncated accretion disc, which is the sum of black-body contributions at different temperatures. We can therefore conclude that for the portions of the light curve that do not show evidence for flaring activity, what is observed is again a combination of the emission from the irradiated companion star and a residual accretion disc. This also explains why no correlation or lag between the NIR and the optical emission can be found in the flat part of the light curves.

A further test for this comes from the independent calculation of the expected $J$-band integrated flux emitted by a G-type companion star, which is irradiated by the spin-down luminosity of an active millisecond radio pulsar and by X-ray luminosity.

To do this, we considered the estimate of the spin-down luminosity $L_{\mathrm{SD}}$ of $\mathrm{J} 1023$ given by Deller et al. (2012) and Archibald et al. (2013), $L_{\mathrm{SD}} \sim 4.4 \times 10^{34} \mathrm{erg} \mathrm{s}^{-1}$. We can then estimate the size of the system using the equations reported in Eggleton (1983) to compute the Roche lobe dimensions, from which we derive an orbital separation $a$ of $1.3 \times 10^{11} \mathrm{~cm}$, i.e. $\sim 1.8 R_{\odot}$, considering a mass of $0.2 M_{\odot}$ for the companion star, and 1.7 $M_{\odot}$ for the NS (Archibald et al. 2009; Deller et al. 2012). We can evaluate the fraction of the spin-down luminosity that is intercepted by the companion star, under the assumption of isotropic emission of the pulsar and considering the typical geometry of an LMXB. In particular, the fraction of the spindown luminosity of the pulsar that is intercepted by the companion star, with a radius equal to its Roche lobe radius $R_{\mathrm{L}_{2}}$, is

$L_{\mathrm{SD}, \text { int }}=L_{\mathrm{SD}} \frac{1-\cos \theta}{2}$,

where $\theta$ is the angular radius of the companion star as seen from the pulsar, i.e. $\theta=\operatorname{arsin}\left(R_{\mathrm{L}_{2}} / a\right)=0.22 \mathrm{rad}$. Therefore, $L_{\mathrm{SD} \text {,int }}=$ $5.28 \times 10^{32} \mathrm{erg} \mathrm{s}^{-1}$, considering an almost negligible albedo.

With a similar reasoning, considering an emitted X-ray luminosity of $7 \times 10^{33} \mathrm{erg} \mathrm{s}^{-1}$ (obtained by converting the measured counts $\mathrm{s}^{-1}$ in our X-ray light curve and considering the known distance of $1.37 \mathrm{kpc}$; Deller et al. 2012), we can also derive the fraction of X-ray luminosity that irradiates the companion, which is $8.4 \times 10^{31} \mathrm{erg} \mathrm{s}^{-1}$. The intrinsic luminosity $L_{*}$ of the G-type star can instead be evaluated starting from the surface temperature of the companion reported in Thorstensen \& Armstrong (2005), i.e. $5700 \mathrm{~K}$. Using the Stefan-Boltzmann relation, $L_{*}=6.32 \times$ $10^{32} \mathrm{erg} \mathrm{s}^{-1}$. By summing these three luminosities, we obtain a maximum luminosity of the star of $1.24 \times 10^{33} \mathrm{erg} \mathrm{s}^{-1}$, which corresponds to a surface temperature of $\sim 6650 \mathrm{~K}$. When we integrate the black-body function for this temperature in the $J$ band, we obtain a black-body flux of $\sim 4 \times 10^{-13} \mathrm{erg} \mathrm{cm}^{-2} \mathrm{~s}^{-1}$, which is consistent with the flux that we measure in the flat part of our $J$-band light curve (i.e. $\sim 3 \times 10^{-13} \mathrm{erg} \mathrm{cm}^{-2} \mathrm{~s}^{-1}$ ). Therefore, we can safely conclude that what we observe in the flat part of the NIR light curve is the NIR emission of the irradiated companion star alone, which is not expected to lag the optical emission, as observed. We caution, however, that several approximations were made for this calculation. As a consequence of this, we would expect to observe an orbital modulation of the NIR light curve. While a modulation is observed in the $B$-band light curve, this is not apparent in the $J$ band. We note, however, that the $B$-band semi-amplitude that we measure is rather low, of the order of $10 \%$. This is lower than what was observed in Coti Zelati et al. (2014), where a $g$-band semi-amplitude of the $40 \%$ was detected. Assuming a scaling with the wavelength, the $J$-band expected semi-amplitude should be of the order of $5 \%$, that is, comparable to the statistic uncertainty of our NIR dataset, suggesting that a low-level modulation is indeed present, although hidden in the uncertainty of our NIR dataset.
Switching to the red CCF in Fig. 6, that is, to the CCF that is relative to the portion of the NIR light curve where flaring activity is detected, we observe a narrow peak at $10 \mathrm{~s} \mathrm{lag,} \mathrm{which} \mathrm{indi-}$ cates that the NIR is delayed with respect to the optical with a lag of $\sim 10 \mathrm{~s}$. To investigate the origin of this positive lag, we first considered the hypothesis that the NIR emission might be the result of reprocessing of the optical emission coming from the accretion disc at the surface of the companion star, as suggested in Shahbaz et al. (2018). According to Bogdanov et al. (2015), the optical variability closely matches the X-ray variability (at least in terms of flare emission, which the authors interpreted as phenomena that span from optical to at least X-ray frequencies), and therefore any X-ray flare should in principle give rise to a corresponding flare in the NIR. Because we lack high timeresolution X-ray observations of the target that are contemporaneous to our NIR and optical data sets, we decided to consider the X-ray $0.3-10 \mathrm{keV}$ variations reported in Bogdanov et al. (2015) to prove this hypothesis, under the assumption that the $\mathrm{X}$-ray variations in the light curves are similar enough in different epochs. These variations are on average of $\sim 30$ counts s$^{-1}$, which, considering a power-law model as X-ray spectrum, corresponds to an unabsorbed flux of $\sim 4.5 \times 10^{-11} \mathrm{erg} \mathrm{s}^{-1} \mathrm{~cm}^{-2}$. Considering the known distance of PSRJ1023 (1.37 kpc), we evaluated a luminosity of $\sim 1 \times 10^{34} \mathrm{erg} \mathrm{s}^{-1}$, which, considering an orbital separation of $\sim 1.8 R_{\odot}$, translates into a flux of $\sim 5 \times 10^{10} \mathrm{erg} \mathrm{cm}^{-2} \mathrm{~s}^{-1}$ that irradiates the companion star. When we multiply this result for the area of the companion star that is irradiated, we obtain the luminosity that is absorbed by the companion star, which is $1.2 \times 10^{32} \mathrm{erg} \mathrm{s}^{-1}$. Adopting an almost negligible albedo $\eta \sim 0.1$, and using the Stefan-Boltzmann relation with a surface temperature of the companion star of $\sim 6650 \mathrm{~K}$ (which we just evaluated considering the spin-down luminosity and the X-ray luminosity as sources of irradiation), we obtain that a flux variation like this in the X-rays would increase the surface temperature of the companion star to $\sim 7080 \mathrm{~K}$. Integrating the black-body Planck function for these two temperatures over the $J$-band filter wavelengths, we finally obtain that the expected flux variation due to reprocessing of the X-ray to optical flaring emission in this band should be of a factor 1.16. However, the amplitude of the flares that we see in our NIR light curve is higher, with a fractional flux variation of a factor of $\sim 1.75$. The X-ray band variability of PSR J1023 is therefore not sufficient to explain the NIR flares that we observe. Together with the clear evidence (see Fig. 2) that no optical or X-ray flare is observed during the HAWK-I observations, this permits to exclude a reprocessing origin of the NIR light curve (at least another component has to be invoked). Moreover, the $\sim 10 \mathrm{~s}$ lag that we observe in our CCF is too high to support a reprocessing origin of the NIR emission because the $1.8 R_{\odot}$ orbital separation is consistent with a lag of $\sim 4 \mathrm{~s}$ only (as reported in Shahbaz et al. 2018). Therefore, the origin of the NIR flaring seems to be different from that of the optical to X-ray flares (which are not detected in our light curves, but are observed e.g. in Papitto et al. 2019 using OM data) for J1023.

Different scenarios might be considered to explain the lag that we observe between the NIR and optical light curves. Bogdanov et al. (2018) observed the emission of radio flares from the system, which the authors interpreted as due to the emission of collimated jets in correspondence with the low X-ray state. The spectrum of the radio emission is found to be flat or slightly inverted at the lowest frequencies, which is a typical signature of the emission of synchrotron radiation from jets. Jets from NS X-ray binaries have mostly been observed in X-ray 
binaries in outburst or in persistent systems (Migliari \& Fender 2006; Russell et al. 2007; Russell \& Fender 2008; Migliari et al. 2010; Baglio et al. 2016b; Tudor et al. 2017). This means that we lack extensive literature data for jets that are emitted in quiescence or in the particular intermediate state in which J1023 lingers. Several cross-correlation studies between X-ray, optical, and NIR light curves have been performed for black hole $(\mathrm{BH})$ X-ray binaries (see e.g. the results for GX 339-4 and V404 Cyg: Casella et al. 2010; Kalamkar et al. 2016; Gandhi et al. 2017; Vincentelli et al. 2018). For these systems, the NIR has always been found to be delayed with respect to the X-ray emission, with a typical lag of $\sim 0.1 \mathrm{~s}$. This lag has been explained in terms of the strongly variable accretion flow that emits in the $\mathrm{X}$-rays, which is able to inject velocity fluctuations at the base of the jet, driving internal shocks at large distances from the BH (Malzac 2013, 2014; Drappeau et al. 2017; Malzac et al. 2018). At the shocks, leptons are accelerated, giving rise to variable synchrotron emission, which should be observed in the radio or NIR. However, according to this model, delays between $\mathrm{X}$-rays and NIR of $\sim 0.1 \mathrm{~s}$ are expected, as is also supported by the observations. The delay measured for PSR J1023 is $\sim 100$ times higher, therefore this scenario seems to be excluded.

Another intriguing possibility is instead that the NIR emission might be delayed with respect to the optical due to reprocessing of the optical emission in a region that is found outside the system itself (if the optical signal propagates at the speed of light, a delay of $10 \mathrm{~s}$ implies a distance of $\sim 3 \times 10^{11} \mathrm{~cm}$ from the region of the optical emission, i.e. $\sim 4 R_{\odot}$, which has to be outside the system $)^{1}$. The recent observations reported in Papitto et al. (2019) seem to suggest that a rotation-powered pulsar may always be active in the system, even if the source is in its accretion state. In the high mode, the relativistic wind emitted from the radio pulsar, interacting close to the light cylinder radius with the flow of matter that accretes from the companion star, creates a shock where electrons are accelerated and thus produces synchrotron radiation in the form of optical and X-ray pulsations, which are observed for J1023. If the radio pulsar is on, the momentum that the pulsar radiation exerts onto the matter that reaches the inner Lagrangian point of the system inhibits the accretion process. This might create a stream of matter, which, instead of being accreted onto the compact object, is ejected, and forms a spiral around the entire system. This phase of ejection of matter is called "radio-ejection" phase, and has been postulated to explain the behaviour of other millisecond pulsar binaries such as PSR J1740-5340 (Burderi et al. 2001, 2002). When we consider that the optical emission comes from the light cylinder radius caused by the interaction between the pulsar wind and the internal part of the accretion disc (as suggested in Papitto et al. 2019), the NIR emission might be the reprocessing of the optical emission after the optical photons meet the stream of ejected matter. If this is true, the $10 \mathrm{~s}$ delay would give an idea of the projected distance between the region of the optical emission, which is located at some $10^{2} \mathrm{~km}$ from the pulsar, and the stream of matter, that is, $\sim 4 R_{\odot}$. This hypothesis is also supported by the fact that we do not observe the delay of $10 \mathrm{~s}$ for the whole light curve, which suggests some kind of asymmetric distribution of the flow of matter around the system. In particular, the $10 \mathrm{~s} \mathrm{lag}$ is observed between phase $\sim 0.3$ and $\sim 0.6$, that is, in the first

\footnotetext{
1 We caution that in principle, without knowledge of the angle subtended at the source by Earth and the reprocessing site, it is impossible to firmly constrain the distance between the source and the reprocessor. As an order of magnitude, we can give some approximate estimates, however. The numbers presented in this discussion contain this uncertainty.
}

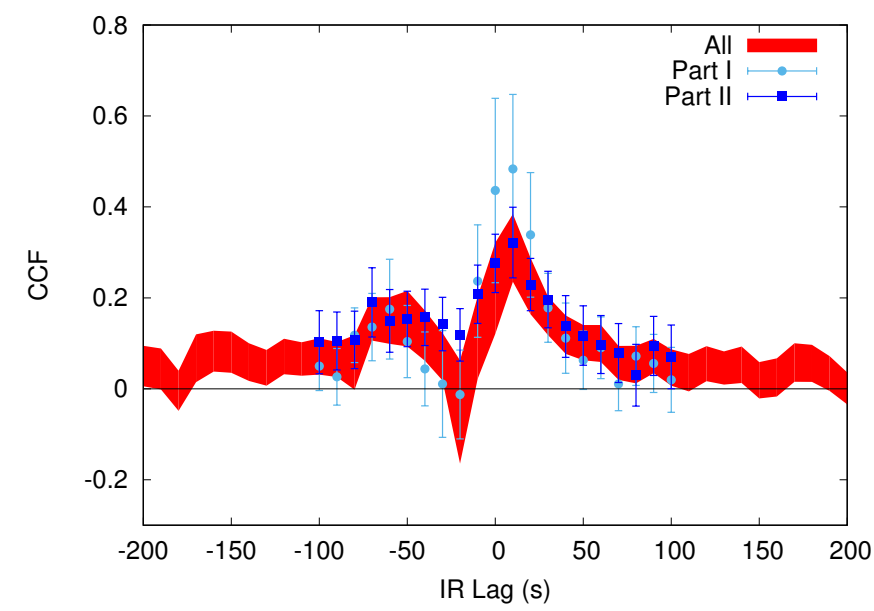

Fig. 8. Optical to NIR CCF obtained in the following two segments: the first $\sim 2000 \mathrm{~s}$ of the observation, and the following $\sim 2500 \mathrm{~s}$ of the observation (light blue dots and blue squares, respectively). The CCF built considering both segments together is superimposed. No significant differences can be reported.

$\sim 5000$ s of the NIR light curve (see Fig. 2). As a test, we tried to compute new CCFs by dividing the flaring part of the NIR light curve into two segments (first segment: the first $\sim 2000 \mathrm{~s}$ of the observation in the $J$-band light curve; second segment: the following $\sim 2500 \mathrm{~s}$ of the observation). No significant differences were found: the peak of the CCFs was always at $\sim 10 \mathrm{~s}$ (see Fig. 8).

\subsection{NIR flares exiting the low mode: evidence of jets or outflows?}

Bogdanov et al. (2018) reported on the detection of radio flares that were emitted in correspondence with the whole duration of an X-ray low mode. During a typical low mode, the radio spectrum of the flare is observed to evolve from being inverted $\left(\alpha \sim 0.4\right.$, where $\left.F_{v} \propto v^{\alpha}\right)$ to steep $(\alpha \sim-0.5)$ over several minutes. This enhanced radio flux, characterized by an evolving radio spectrum, is interpreted as a signature of the launching of an evolving outflow or jet. During the high mode, the radio flux drops by a factor of 3-4 with respect to its level during the low mode. The radio spectrum in this case is on average found to be slightly inverted $(\alpha \sim 0.2)$. The fact that the radio emission is not switched off during the high mode suggests that the outflow is not totally quenched after the transition to the high mode, but a self-absorbed compact jet might still be emitted.

Jets emitted in low-luminosity X-ray binaries such as PSR J1023 are not expected to contribute significantly in the NIR; as shown in Baglio et al. (2016a), no contribution from synchrotron emission of a jet or a generic outflow is observed at NIR frequencies in the broadband SED. If synchrotron radiation is emitted from an outflow during the X-ray low mode, we can therefore hypothesize that the break frequency of its spectrum will be within the radio band, which means that the jet will mainly contribute in the radio. The fact that the radio spectrum of the jet during the low mode is found to evolve from inverted to steep suggests that the jet break frequency might be found already in the radio band (but at a frequency $>12 \mathrm{GHz}$ ) at the beginning of the flare, and then moves to lower frequencies $(<8 \mathrm{GHz})$ while the jet evolves during the low mode. To visualize the evolution of the spectrum, we show in Fig. 9 the possible synchrotron spectrum of the outflow at the start of the low mode 


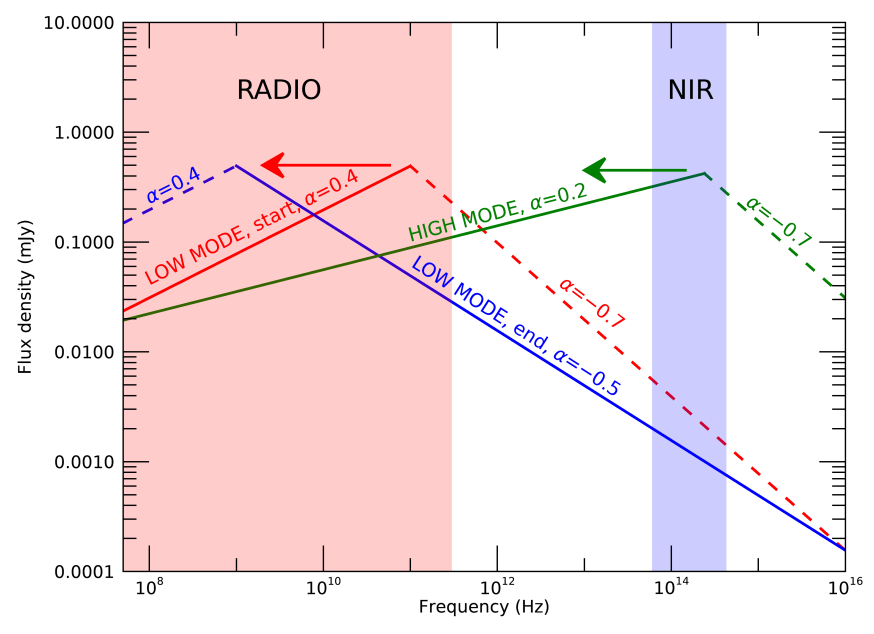

Fig. 9. Representation of the possible evolution of the outflow emission spectrum during the high and the low X-ray mode. In red we show the possible synchrotron spectrum of the outflow at the start of the low mode. In blue we plot the possible spectrum of the outflow at the end of the low mode. In green we show the possible spectrum of the outflow at the beginning of the high mode. All the break frequency values have been chosen arbitrarily to explain the model, but the spectral index values and the normalization (flux) values are based on observations. With two arrows we indicate the plausible direction of the evolution of the break frequency. The red and blue regions of the figure represent the radio and NIR bands, respectively.

in red, using the slope $\alpha=0.4$ measured by Bogdanov et al. (2018) for the self-absorbed part (solid line), and an arbitrary slope $\alpha=-0.7$ for the optically thin part of the spectrum (dashed line), which is typical of jets and outflows in X-ray binaries. In blue we show the possible spectrum of the outflow at the end of the low mode, using the slope $\alpha=-0.5$ measured by Bogdanov et al. (2018) for the optically thin part (solid line) and maintaining the slope $\alpha=0.4$ for the optically thick part (dashed line). After the transition to the high mode, a compact selfabsorbed jet might be launched (Bogdanov et al. 2018). In Fig. 9 the possible spectrum of the outflow in the high mode is shown in green, using the slope $\alpha=0.2$ reported in Bogdanov et al. (2018) for the self-absorbed synchrotron part (solid line) and the typical $\alpha=-0.7$ slope for the optically thin part (dashed line). When at the beginning of the high mode (i.e. when the compact jet is launched) the jet break frequency is in the NIR range, this might give rise to an enhanced NIR flux. We can then assume that the break frequency shifts towards lower frequencies (but remains $>12 \mathrm{GHz}$ ), without changing the radio spectral shape, in accordance with Bogdanov et al. (2018) (see Fig. 9). In this way, we would observe a NIR flare that corresponds to the transition to the high mode. A similar phenomenon was also observed in Papitto et al. (2019), where an infrared flare was possibly detected in correspondence to three low-high transition. In addition, Papitto et al. (2019) did not observe NIR flares when the source entered the low mode; this is compatible with our interpretation of the NIR flare at the beginning of the X-ray high mode as due to the evolving position of the jet-break frequency from higher (NIR) to lower (radio) frequencies during the high mode.

\section{Conclusions}

We reported on a NIR (VLT/HAWK-I, $J$ band, $0.5 \mathrm{~s}$ time resolution), optical (XMM-Newton/OM, B band) and X-ray
(Swift/XRT and XMM-Newton RGS) simultaneous campaign performed on 2017 June 9-10 on the transitional millisecond pulsar PSR J1023+0038. The main results of this campaign are summarized below.

- The optical light curve shows the expected sinusoidal modulation at the source $4.75 \mathrm{~h}$ orbital period; when the sinusoidal modulation is subtracted, no evidence for flaring activity or transitions between the active and passive mode is detected. The NIR light curve instead is found to be extremely variable, with strong flares of $\sim 0.3 \mathrm{mJy}$ amplitude and a duration of several minutes in the first $\sim 5000 \mathrm{~s}$ of the observation, while the rest of the light curve is found to be mostly flat, except for one single strong flare. The X-ray light curve shows one transition from low to high X-ray mode, which is found to occur soon before one of the strongest NIR flares that we detect in the NIR light curve.

- We computed the CCF between the optical and NIR light curves. We divided the NIR and optical light curves into two different segments because the NIR light curve is bimodal, and computed two different CCFs. The CCF corresponding to the flat part of the NIR light curve is flat, and no significant feature is reported. The CCF corresponding to the flaring segment of the curve instead shows a clear peak at $\sim 10 \mathrm{~s}$, which indicates that the NIR is delayed with respect to the optical with a $10 \mathrm{~s}$ lag. The power density spectrum shows a decreasing power-law trend with index $\sim-1.2$, which typically indicates some aperiodic activity in X-ray binaries.

- We tentatively interpret the $10 \mathrm{~s}$ lag between optical and NIR as due to reprocessing of the optical emission at the light cylinder radius with a stream of matter that spirals around the system after it was ejected in the radio-ejection phase. This interpretation is strongly supported by the high time delay, which is a clear indication that the optical emission cannot be reprocessed in a region inside the system.

- We observed a NIR flare in correspondence to the transition from the low to the high X-ray mode of the source. The interpretation of this feature is non-trivial. We hypothesize that the flare might be due to the emission of a jet or a noncollimated outflow, as has been suggested by Bogdanov et al. (2018). According to our interpretation, the infrared flare would be due to the evolving spectrum of the jet or outflow, the jet-break frequency moving rapidly from higher (NIR) to lower (radio) frequency after the launching of the jet, which has to occur at the low to high mode transition.

Acknowledgements. The research reported in this paper is based on observations made with ESO Telescopes at the Paranal Observatory under programme ID 299.D-5023 (PI: Campana). PDA and SC acknowledge support from ASI grant $\mathrm{I} / 004 / 11 / 3$. A.P. acknowledges financial support from the Italian Space Agency and National Institute for Astrophysics, ASI/INAF, under agreements ASI-INAF I/037/12/0 and ASI-INAF n.2017-14-H.0. We thank the anonymous referee for useful comments that helped us to improve the paper.

\section{References}

Alpar, M. A., Cheng, A. F., Ruderman, M. A., \& Shaham, J. 1982, Nature, 300, 728

Ambrosino, F., Papitto, A., Stella, L., et al. 2017, Nat. Astron., 1, 854

Archibald, A. M., Stairs, I. H., Ransom, S. M., et al. 2009, Science, 324, 1411

Archibald, A. M., Kaspi, V. M., Hessels, J. W. T., et al. 2013, ArXiv e-prints [arXiv:1311.5161]

Archibald, A. M., Bogdanov, S., Patruno, A., et al. 2015, ApJ, 807, 62

Baglio, M. C., D’Avanzo, P., Campana, S., et al. 2016a, A\&A, 591, A101

Baglio, M. C., D'Avanzo, P., Campana, S., et al. 2016b, A\&A, 587, A102

Bogdanov, S., Archibald, A. M., Bassa, C., et al. 2015, ApJ, 806, 148

Bogdanov, S., Deller, A. T., Miller-Jones, J. C. A., et al. 2018, ApJ, 856, 54 
Bond, H. E., White, R. L., Becker, R. H., \& O’Brien, M. S. 2002, PASP, 114, 1359

Burderi, L., Possenti, A., D’Antona, F., et al. 2001, ApJ, 560, L71

Burderi, L., D’Antona, F., \& Burgay, M. 2002, ApJ, 574, 325

Campana, S., Coti Zelati, F., Papitto, A., et al. 2016, A\&A, 594, A31

Casella, P., Maccarone, T. J., O’Brien, K., et al. 2010, MNRAS, 404, L21

Coti Zelati, F., Baglio, M. C., Campana, S., et al. 2014, MNRAS, 444, 1783

Coti Zelati, F., Campana, S., Braito, V., et al. 2018, A\&A, 611, A14

Deller, A. T., Archibald, A. M., Brisken, W. F., et al. 2012, ApJ, 756, L25

Deller, A., Degenaar, N., Hessels, J., et al. 2015, ATel, 7255

den Herder, J. W., Brinkman, A. C., Kahn, S. M., et al. 2001, A\&A, 365, L7

Dhillon, V. S., Marsh, T. R., Stevenson, M. J., et al. 2007, MNRAS, 378, 825

Drappeau, S., Malzac, J., Coriat, M., et al. 2017, MNRAS, 466, 4272

Eggleton, P. P. 1983, ApJ, 268, 368

Gandhi, P., Bachetti, M., Dhillon, V. S., et al. 2017, Nat. Astron., 1, 859

Hakala, P., \& Kajava, J. J. E. 2018, MNRAS, 474, 3297

Hernandez Santisteban, J. V. 2016, PhD Thesis, University of Southampton Jaodand, A., Archibald, A. M., Hessels, J. W. T., et al. 2016, ApJ, 830, 122

Kalamkar, M., Casella, P., Uttley, P., et al. 2016, MNRAS, 460, 3284

Kissler-Patig, M., Pirard, J.-F., Casali, M., et al. 2008, A\&A, 491, 941

Li, J.-Y., Samarasinha, N. H., Kelley, M. S. P., et al. 2014, ApJ, 797, L8

Malzac, J. 2013, MNRAS, 429, L20

Malzac, J. 2014, MNRAS, 443, 299

Malzac, J., Kalamkar, M., Vincentelli, F., et al. 2018, MNRAS, 480, 2054
Migliari, S., \& Fender, R. P. 2006, MNRAS, 366, 79

Migliari, S., Tomsick, J. A., Miller-Jones, J. C. A., et al. 2010, ApJ, 710, 117 Papitto, A., \& Torres, D. F. 2015, ApJ, 807, 33

Papitto, A., Ferrigno, C., Bozzo, E., et al. 2013, Nature, 501, 517

Papitto, A., Rea, N., Coti Zelati, F., et al. 2018, ApJ, 858, L12

Papitto, A., Ambrosino, F., Stella, L., et al. 2019, ApJ, 882, 104

Radhakrishnan, V., \& Srinivasan, G. 1982, Curr. Sci., 51, 1096

Russell, D. M., \& Fender, R. P. 2008, MNRAS, 387, 713

Russell, D. M., Fender, R. P., \& Jonker, P. G. 2007, MNRAS, 379, 1108

Shahbaz, T., Linares, M., Nevado, S. P., et al. 2015, MNRAS, 453, 3461

Shahbaz, T., Dallilar, Y., Garner, A., et al. 2018, MNRAS, 477, 566

Srinivasan, G. 2010, New A Rev., 54, 93

Stappers, B. W., Archibald, A. M., Hessels, J. W. T., et al. 2014, ApJ, 790, 39

Szkody, P., Fraser, O., Silvestri, N., et al. 2003, AJ, 126, 1499

Takata, J., Li, K. L., Leung, G. C. K., et al. 2014, ApJ, 785, 131

Tendulkar, S. P., Yang, C., An, H., et al. 2014, ApJ, 791, 77

Thorstensen, J. R., \& Armstrong, E. 2005, AJ, 130, 759

Tudor, V., Miller-Jones, J. C. A., Patruno, A., et al. 2017, MNRAS, 470, 324

Uttley, P., McHardy, I. M., \& Vaughan, S. 2005, MNRAS, 359, 345

van der Klis, M. 1988, in Timing Neutron Stars, eds. H. Ogelman, \& E. P. J. van den Heuvel (Dordrecht: Kluwer), NATO ASI Series C, 262, 27

Vincentelli, F. M., Casella, P., Maccarone, T. J., et al. 2018, MNRAS, 477, 4524

Wijnands, R., \& van der Klis, M. 1998, ATel, 17, 1

Zampieri, L., Burtovoi, A., Fiori, M., et al. 2019, MNRAS, 485, L109 